\title{
Framework for reversible data hiding using cost-effective encoding system for video steganography
}

\author{
Manjunath Kamath K. ${ }^{1}$, R. Sanjeev Kunte ${ }^{2}$ \\ ${ }^{1}$ Department of Information Science and Engineering, Yenepoya Institute of Technology, India \\ ${ }^{2}$ Department of Computer Science and Engineering, J.N.N College of Engineering, India
}

\begin{tabular}{l}
\hline \hline Article Info \\
\hline Article history: \\
Received Oct 24, 2019 \\
Revised Apr 23, 2020 \\
Accepted May 4, 2020 \\
\hline
\end{tabular}

Keywords:

Blocking

Data security

Encoding

Intra-prediction

Reversible data hiding

\begin{abstract}
Importance's of reversible data hiding practices are always higher in contrast to any conventional data hiding schemes owing to its capability to generate distortion free cover media. Review of existing approaches on reversible data hiding approaches shows variable scheme mainly focusing on the embedding mechanism; however, such schemes could be furthermore improved using encoding scheme for optimal embedding performance. Therefore, the proposed manuscript discusses about a cost-effective scheme where a novel encoding scheme has been used with larger block sizes which reduces the dependencies over larger number of blocks. Further a gradient- based image registration technique is applied to ensure higher quality of the reconstructed signal over the decoding end. The study outcome shows that proposed data hiding technique is proven better than existing data hiding scheme with good balance between security and restored signal quality upon extraction of data.
\end{abstract}

Copyright $@ 2020$ Institute of Advanced Engineering and Science. All rights reserved.

\section{Corresponding Author:}

Manjunath Kamath K.,

Department of Information Science and Engineering,

Yenepoya Institute of Technology,

Moodbidri, India.

Email: kamathsjce@gmail.com

\section{INTRODUCTION}

With the increasing threats of data stealing and tampering by the adversaries, the process of data hiding is gaining importance. According to the conventional mechanism, data hiding deals with the mechanism of hiding sensitive information within a cover data of any form [1,2]. It will mean that conventional data hiding process refers to using two types of data viz. i) a secret data or message which needs to be covertly forwarded to receiver and ii) a cover data which is visible for anyone. However, the connectivity of these two types of data is often used in different ways. For an example, the secret message is quite irrelevant with respect to the cover data in tactical and covert communication system. However, authentication mechanism demands the correlation of the secret message to be embedded is high with respect to the cover data. A closer look in both the type of application tells that invisibility of the secret message is significantly important [3].

Another characteristics of conventional data hiding is that incorporation of any form of mechanism will lead to some sort of distortion over the cover data as the secret message is hidden within it. This also results in failure in extracting the secret message from the original media [4]. It will eventually mean that cover media has underwent to permanent distortion post the process of hiding the secret message. However, there are some sectors of application (e.g. law enforcement, medical diagnosis, etc.) that always demands target data to be reversible to the original source data (cover data) even after data is hidden with it are extracted for some reason. Apart from this, various other applications (investigation of high-energy particle and remote sensing, etc) demands recovery of the actual cover data owing to demands of accuracy. The operations that deals with these approaches are often termed as lossless or reversible data hiding [5, 6]. 
The concept of reversible data hiding approach offers benefits for different ranges of applications that connects both this set of data in such a way that it is feasible to extract the cover data losslessly post the extraction of the secret message. Unfortunately, majority of the conventional data hiding practices does not deal with reversibility factor.

The process of reversible data hiding technique basically consists of two steps e.g. i) embedding the secret message over cover data and ii) extracting the secret message from the cover data. There is also a dependency of secret key in order to perform the embedding and extraction process. The proposed system deals with a reversible data hiding process consider image as secret message and video as cover data. Accordingly, the host video content will be subjected to the data embedding system within which the secret message in terms of image will be embedded. Upon performing successful embedding process, the next step will be to perform data extraction where the system should extract the secret message (image) from the encoded cover media (video). One of the significant motivating factors for adopting reversible data embedding system is that it is independent of any form of embedding process with distortion.

At present, there are various conventional approaches for performing reversible data hiding technique [7-9]. Some of the significant approaches are substitutions using least significant bits, difference expansion, and histogram modification. All these approaches do have advantages as well as reporting limitations also. Therefore, the clear statement of the problem of the proposed study will be to design a reversible data hiding framework overcoming the limitation of existing system to improve the embedding performance. Therefore, the proposed system introduces a cost-effective approach where both security as well as computational efficiency of reversibility factor of the data hiding scheme is retained to its maximum level. The organization of the proposed paper is as follows: Section 1 discusses about the existing literatures where different techniques are discussed for detection schemes used in power transmission lines followed by discussion of research problems and proposed solution. Section 2 discusses about algorithm implementation followed by result analysis in Section 3 and conclusive remarks are provided in Section 4.

At present there are various studies focusing on reversible data hiding practices, some of which are updated in our prior work [10]. The recent work of Wang et al. [11] have used block truncation coding using absolute mean along with encryption using chaos theory with a claim of improved security robustness. Adoption of histogram modification is another frequently used approach for enhancing the contrast level of an image as the process of reversible data hiding (Wu et al. [12]). The work of Peng et al. [13] applies the encryption process by a service provider who performs ciphering of already ciphered data by user using the concept of reversible mapping model. The work of Puteaux and Puech [14] has used most significant bits of increasing the embedding capacity using predictive approach. Existing system has also witnessed usage of ciphering process over image bitstreams using reversible data hiding approach as seen in work of Qian et al., [15].

Adoption of binary tree with discrete labeling approach was also proven to improve the performance of reversible data hiding approach [16]. Homomorphic encryption is also claimed of offering better encryption performance over multimedia data [17]. Jiang et al., [18] have used encryption of the bitstreams using least significant bits over a mesh model for performing reversible data hiding. Xiang and Luo [19] have also Paillier encryption system with a better option for resisting pixel oversaturation that has beneficial aspect over the performance of data hiding approach. A transformation-based approach was used by Zhang et al., [20] where the information of the source image is transformed to another image with supportability of lossless restoration process. Existing approach also used multiplexing approach associated with the code division by Ma and Shi [21] along with Walsh Hadamard. Qian and Zhang [22, 23] have used low density parity check over the series of the selected bits using Slepian wolf code. The authors have also performed encryption of the bitstreams over the conventional JPEG format.

The capacity of the conventional reversible data hiding was subjected to improvement using sparse representation as seen in the work of Cao et al. [24]. Qiu et al. [25] have presented an adaptive scheme where integer transformation scheme has been used for improving the embedding efficiency in reversible data hiding. Consideration of multiple numbers of predictors is also proven to improve the performance of reversible data hiding as seen in work of Jafar et al. [26]. Optimal modification of the histogram is considered in the work of $\mathrm{Hu}$ et al. [27] where a pixel prediction-based approach is used with approximation of the embedding performance. Li et al. [28] have used a histogram-sequence based technique where histogram modification is used for for improving the embedding performance. Another implementation of histogram modification-based approach was used by Li et al. [28] where multiple numbers of histograms are selected. The work of Zhou et al. [29] has used supervised learning mechanism for differentiating the patches of ciphering and non-ciphering as the process of feature extraction. Therefore, there are various existing studies focusing on improving the performance of reversible data hiding approach. The next section discusses about research problems that has been identified from the existing approaches of reversible data hiding techniques. 
The significant research problems are as follows:

- $\quad$ Majority of the existing approaches have used histogram modification without considering the complex forms of frames in videos, where practical applicability of encoding reduces.

- $\quad$ Study towards computational complexity associated with the any existing approach of reversible data hiding is not discussed.

- $\quad$ Adoption of multiple forms of secret images over a video as a cover image and their relationship has never been studied till date.

- Adoption of motion compensation for ensuring better rate distortion performance while performing reversible data hiding is yet to be explored over dynamic scenarios.

Therefore, the problem statement of the proposed study can be stated as "Developing a cost-effective mechanism to perform reversible data hiding process with better embedding performance and ensuring lightweight operation of securing data over video cove file".

The proposed work is an extension of our prior work [10] that has discussed about the video steganography. The proposed scheme introduces a simplified and cost-effective approach of reversible data hiding approach where the prime emphasis is given for embedding and encoding process for better performance. The adopted scheme of implementation of proposed system is shown in Figure 1.

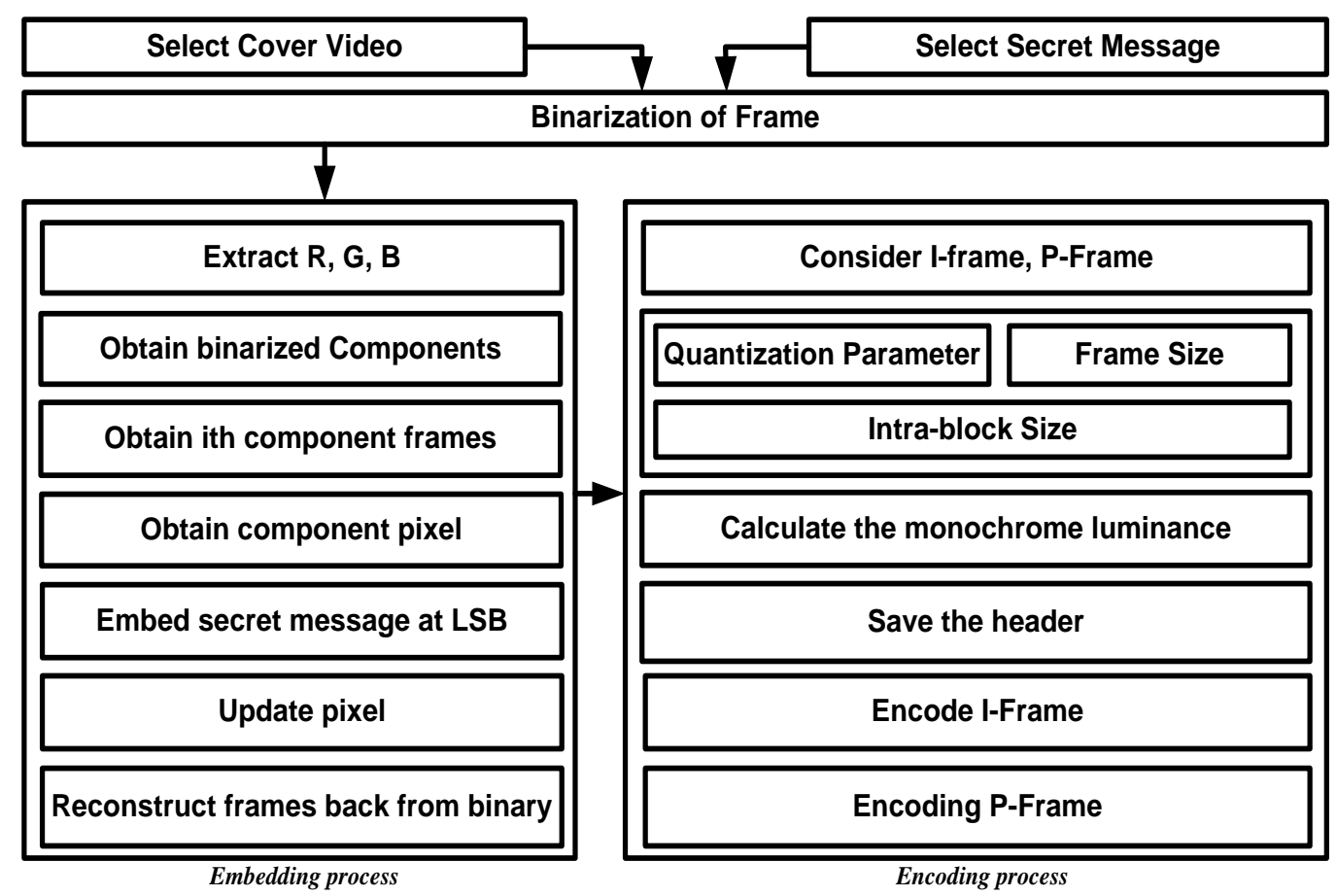

Figure 1. Research methodology of proposed system

The implementation is carried out using analytical research methodology where the video source is considered as a cover file while a secret image is considered as a data to be embedded within the video cover file. Both the input sources are subjected to binarization followed by embedding process where the red, green, and blue color components are extracted for obtaining binarized component, $\mathrm{i}^{\text {th }}$ component, and others associated component pixel. The secret message is embedded using least significant bits followed by updating of the component pixels and finally the reconstructed frames are regained back from the binary components. This operation is further followed by considering I and $\mathrm{P}$ frame where further three inputs are considered viz. quantization parameter, frame size, and intra-block size. The next part of the implementation is about calculating the monochrome luminance which is further followed by final encoding operation. All the I-frame as well as P-Frame are finally encoded. The significant contribution of the proposed system is that it considers larger block of frames for performing encoding process which is further extended to use a tree-structured motion estimation approach as a part of block partitioning process. This operation makes the data hiding process much more comprehensive without any significant loss of data quality of the reconstructed signal as well as secret data.

Framework for reversible data hiding using cost-effective encoding system for ... (Manjunath Kamath K.) 


\section{SYSTEM DESIGN}

The proposed system introduces a unique process of reversible data hiding scheme by emphasizing the secure data transmission over wireless network with retention of maximum information. The prime research challenge is to ensure that while performing embedding of the secret data, there should not be significant loss of the video cover. Although, there are certain application where cover medium is absolutely not subjected to any form of degradation e.g. military, medical, and forensic, etc. The proposed system introduces a novel mechanism of reversible data hiding scheme where the pixel values are subjected to alteration for embedding the secret data within a video file. The prime objective of this system design is to ensure better and cost-effective secured communication. A contributory part of the implementation is its unique recovery process of the secret data from the video without any dominant traces of the distortion. This section discusses about the implementation scheme adopted in proposed system with respect to system assumption, implementation strategy, and execution flow.

\subsection{System assumption}

The primary assumption of the proposed system is that the both the secret data to be hidden and the source video, acting as a cover file, is untampered and retains its genuine form. The video file is considered to be the uncompressed multimedia file that is subjected to further encoding process. The secondary assumption of the proposed study is that it offers significant robustness, perceptibility, security, and capacity in order to offer better form of reversible data hiding technique. The tertiary assumption of the proposed study is that the transmission of the hidden and embedded data is carried out by a transmitter that is operating under a wireless environment.

\subsection{Implementation strategy}

The complete implementation strategy of the proposed reversible data hiding scheme is carried out based on proposed encoding mechanism that consider all the positive and negative aspect of variable block size. The prime contradiction is that a smaller block size offers better reduction to the probability of presence of multiple motions over a block. Similarly, higher block size offers advantage of controlling the overhead associated with partitioning process and motion vector. Therefore, the proposed system integrates these two facts of block size in order to enhance the trade-off between distortion and rate. The proposed scheme offers selection of macro-block of higher order sizes and not the smaller sizes (e.g. 16x16) that is originally defined in conventional H.264 protocol. The selected macroblock is further classified to smaller sizes of blocks e.g. $4 \times 4$ or $8 \times 8$. This adoption of strategy benefits the reversible data hiding process significantly as it minimizes the bit rate of the frame as the homogeneous regions within the frame are subjected to coding using a smaller number of blocks only. Therefore, the proposed system adopts larger block only as it minimizes the dependencies of higher number of blocks that are required to be compensated of any motion and hence it minimizes processing time to large extent. The motion compensation scheme used in proposed reversible data hiding scheme can be empirically represented as,

$$
\left[\mathrm{x}_{2} \mathrm{y}_{2}\right] \rightarrow\left[\left(\mathrm{x}_{1}+\varphi_{1}\right)\left(\mathrm{y}_{1}+\varphi_{2}\right)\right]
$$

In the (1), the study considers that there are two blocks say $\mathrm{B}_{1}\left(\mathrm{x}_{1} \mathrm{y}_{1}\right)$ and $\mathrm{B}_{2}\left(\mathrm{x}_{2} \mathrm{y}_{2}\right)$. In the above expression, the variable $\varphi_{1}$ and $\varphi_{2}$ represents a sophisticated mapping function. The variable $\varphi_{1}$ is computed considering product of basis function as well as motion parameters with respect to the available parameters of motion. The construction of the basis function $\mu$ is carried out considering the discrete cosine function in order to offer the motion of non-rigid order as it can reduce the number of coefficients.

$$
\mu\left(\mathrm{x}_{1} \mathrm{y}_{1}\right) \rightarrow \cos \psi_{1}+\cos \psi_{2}
$$

In the (2), the basis function is shown with respect to blocks of both vertical and horizontal dimensions. The next part of the implementation of the encoding mechanism will be to perform necessary estimation of the motion parameters that will render the most optimal block prediction for a given reference frame. There are various studies that shows that gradient-based image registration approach for the purpose of estimating such motion parameters. The prime contribution of proposed system is that it mechanizes the gradient-based image registration approach for reducing the error score between two blocks $\mathrm{B}_{1}\left(\mathrm{x}_{1} \mathrm{y}_{1}\right)$ and $\mathrm{B}_{2}\left(\mathrm{x}_{2} \mathrm{y}_{2}\right)$. Mathematically, it is computed as,

$$
\text { Error }=\left[\mathrm{B}_{1}\left(\mathrm{x}_{1} \mathrm{y}_{1}\right)-\mathrm{B}_{2}\left(\mathrm{x}_{2} \mathrm{y}_{2}\right)\right]^{\mathrm{n}}
$$


In the (3), the error computation is carried out considering $n$ number of image pixels over the area where there is an overlapping of two image blocks considering intensity of image pixels. The motion estimation of the proposed system is carried out using a unique graphical structure that offers extensive operation to the encoding process while dealing with both uniform and non-uniform regions of the frame in proposed reversible data hiding process. The presence of larger block size in the bottom level of the graph is always an advantageous feature with very less options of motion. On the other hand, the multiple partitioning methods adopted in proposed system offers comprehensive active regions within the frames that are characterized by complex motion vector. Assume that a video frame is considered with respective division of the macro-blocks considering $i$ ) a minimum size of block in the form of integer and $i$ ) cumulative number of partitions. There are multiple mechanisms of splitting this macro-block in different number of ways based on these parameters. Hence, a computationally cost-effective encoding technique is introduced in proposed system with better stability in reversible data hiding performance.

\subsection{Execution flow}

The primary step of the proposed system is to take the input of raw and uncompressed video file. The input video is considered within a range from start frame $\left(\mathrm{s}_{\mathrm{f}}\right)$ to end frame $\left(\mathrm{e}_{\mathrm{f}}\right)(\mathrm{Line}-1)$. For the purpose of investigation, the analysis has been carried out till selected $e_{f}$ value. The input frame is resized suitably followed by applying a method $g_{1}(\mathrm{x})$ on the input video vid in order to yield a matrix $f$ that stores all the selected frames (Line-2). The next part of the implementation is about selecting a data $m s g$ that is subjected to reversible data hiding technique in proposed system (Line-3). The core part of the implementation towards embedding secret data $m s g$ within the video in the form of frame $f$. The execution steps of the proposed algorithm are as follow:

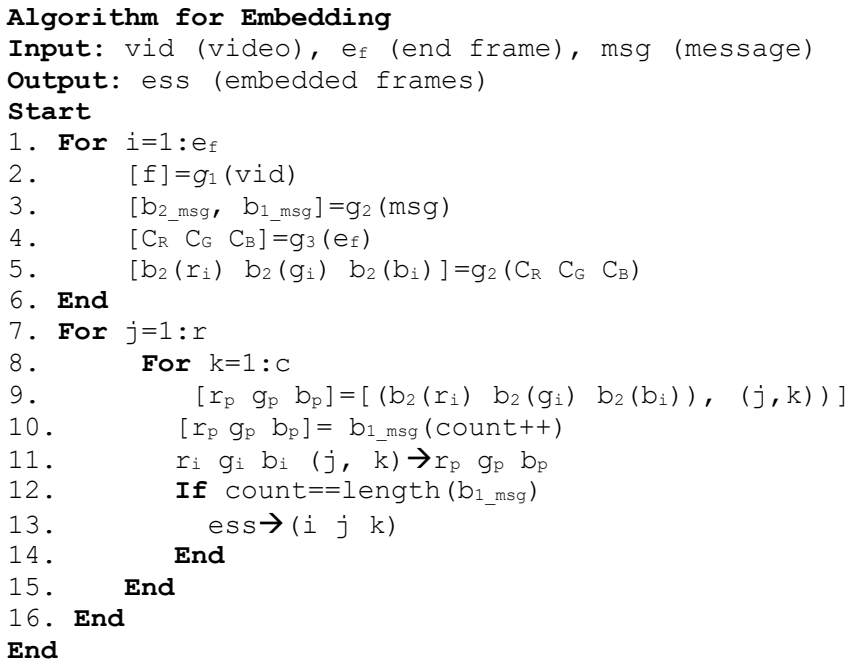

The core part of the algorithm implementation is basically a function $g_{3}(\mathrm{x})$ that mainly performs the embedding operation over the end frame $\mathrm{e}_{\mathrm{f}}$ (Line-4). The function $g_{3}(\mathrm{x})$ extracts the rows and column size from the selected frame and subject it to the transformation for all the image pixels to binary sequence of 8 bits. The extraction is carried out for distinct components of red, green, and blue $C_{R}, C_{G}$, and $C_{B}$ respectively. The similar function $\mathrm{g}_{2}(\mathrm{x})$ is also implemented in next step for binary transformation of the frames (in order to obtain a long binary sequence) for each of the colored components (Line-5). This operation leads to generation of individual binary component of each respective colors i.e. $b_{2}\left(r_{i}\right), b_{2}\left(g_{i}\right)$, and $b_{2}\left(b_{i}\right)$ respectively (Line-5). The constructing a matrix is carried out for $\mathrm{i}^{\text {th }}$ red, green, and blue frame. The implementation considers total number of rows $r$ and column $c$ for $\mathrm{i}^{\text {th }}$ red frames and performs embedding operation of the secret data $m s g$ (Line-12). For this purpose, a temporary matrix is constructed for each colored pixels viz. red pixel $r_{p}$, green pixel $g_{p}$, and blue pixel $b_{p}$ respectively (Line-9). The algorithm remodels the matrix of respective binary message in increasing order count over the red, green, and blue pixels (Line-10). The proposed system extracts the new value of $i, j$, and $k$ to be essential elements when the number of count is equivalent to length of the elements of the binary secret message. All the respective elements of new $\mathrm{i}^{\text {th }}$ red, green, and blue frames are collected (Line-11). Finally, all the essential frames (ess) are collected (Line-13).

The next part of the implementation is associated with the encoding operation of the frames using three different variables viz. i) quantization parameter, ii) specific frame size, and iii) size of intra block. The proposed system primarily performs initializing the indexing process. For all the selected range of frames, the proposed system extracts all the frames first from the video followed by resizing of the frames.

Framework for reversible data hiding using cost-effective encoding system for ... (Manjunath Kamath K.) 
The monochrome luminance is computed followed by configuring the encoding parameters viz. height and width of frames, size of macroblocks for $\mathrm{P}$ frame that is further switched for extended motion estimation. The size of the input video sequence is considered followed by saving the header which consists of encoded bits for each parameter.

This operation generates a bitstream with an input argument of height, width, quantization parameter, start frame, end frame, and size of block thereby assisting better encoding of theI frame. The encoding operation has been carried out using the motion compensation prediction-based approach in order to obtain a better degree of performance for reversible data hiding scheme. This scheme is very different from existing system that doesn't emphasize more on the performance of rate distortion. The proposed system introduces a novel encoding scheme that extends the existing prediction scheme by including prediction of motion to non-translation form. The study uses a two-dimension cosine-based function using elastic model in order to compute the motion of non-translation order between all the blocks. A larger set of blocks has been considered in the proposed encoding scheme with partitioning of higher level in order to achieve better performance in data hiding process.

After all the headers are saved, the encoding of the I-frame is initiated, and this is followed by appending all the I-frame to the headers. The I-frames are then extracted followed by the encoding operation. All the received frames are then stored followed by appending all the I-frame to the bitstreams and generation of the reference frame for P-frame. Similar operation is also carried out for P-frame. The bitstream is then forwarded to the receiver where the decoding operation is continued.

Apart from this, the proposed system also enhances data hiding performance using coding of intra-frames for exploring the optimal outcome of the pixel blocks within the current frame that is considered to be residing within the reference frame. The study considers reference frame to be the frame that has been forwarded by the transmitter to the receiver in prior round of reversible data hiding operation. In order to carry out this operation, the proposed system takes a bigger size of macro-block. The approach computes motion vector of translational form that has lower cost (using Lagrangian cost function) followed by computing the parameters of elastic motion followed by assessing the resources involved in performing the motion compensation using Lagrangian cost function.

The condition applied is that if the resource involvement for performing elastic motion is found to be lesser than the translation motion than the system consider the presented elastic motion compensation in case of this mode of partition. Figure 2 highlights the mechanism of splitting the frames into blocks where the cumulative rate and distortion is computed followed by finding the optimal mode with highly reduced cost involvement. The splitting process continues for all the smaller block sizes. This process also ensures that proposed system is highly capable of controlling the computational complexity.

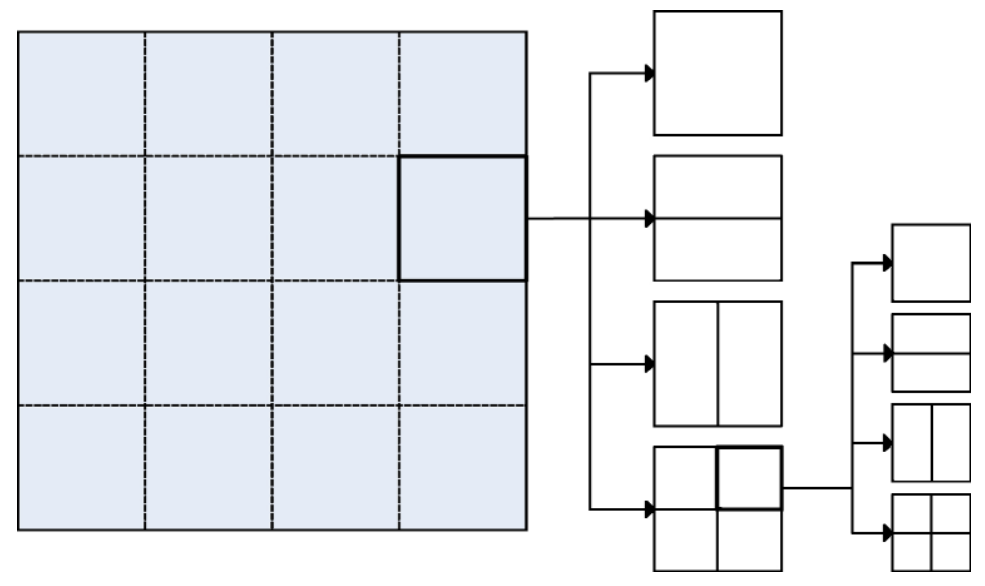

Figure 2. Splitting process of frames to blocks in proposed system

\section{RESULTS ANALYSIS}

The implementation of the proposed system is carried out considering cover video as raw and uncompressed video files [30] where all the QCIF, CIF, WVGA, and HD formats are available for the video. In order to carry out the analysis, the proposed system considers the secret message to be in the form of image. The study considers three different types of image i.e. standard gray scaled image as shown in Table 1, colored image in Table 2, and image captured from devices in Table 3. The analysis is carried out on 
single range of quantization parameter $(\mathrm{QP}=16)$ over 4 different formats of uncompressed video as well as multiple sizes of videos. The final estimation is carried out for number of bits (NB), number of compressed bits (NCB), and compression ratio (CR). The outcome shows that compression ratio performance is good for real time images as well as there is a significant level of increment of other factors too. These performance parameters have potential connectivity with the concept of reversible data hiding. Basically, the increment of block size will reduce the capacity of hiding. The study outcome shows that NB has increased for real-time frames as well as colored image in average while it doesn't have significant improvement in grayscale image. Similarly, NCB as well as CR is found higher for all the types of an image showing better compression performance while performing reversible data hiding process. As reversible data hiding process will need evidence that host image is exactly similar to reconstructed image and this approximated increase in value of these performance parameters shows that proposed system offers better data reversibility in hiding.

Table 1. Analysis using gray scale image

\begin{tabular}{|c|c|c|c|c|c|}
\hline Frame size & SIB & Video size & N.B & N.C.B & C.R \\
\hline \multirow[t]{3}{*}{ QCIF(144x176) } & 4 & $144 \times 176$ & 1013760 & 272630 & 3.7184 \\
\hline & 8 & $160 \times 192$ & 1228800 & 331682 & 3.7048 \\
\hline & 16 & $128 \times 128$ & 983040 & 264738 & 3.7133 \\
\hline \multirow[t]{3}{*}{ CIF (288x352) } & 4 & $288 \times 352$ & 4055040 & 851384 & 4.7629 \\
\hline & 8 & $288 \times 352$ & 4055040 & 865739 & 4.6839 \\
\hline & 16 & $320 \times 384$ & 4915200 & 1019269 & 4.8223 \\
\hline \multirow[t]{3}{*}{ WVGA(480x800) } & 4 & $480 \times 800$ & 4055040 & 865739 & 4.6839 \\
\hline & 8 & $480 \times 800$ & 15360000 & 2206645 & 6.9608 \\
\hline & 16 & $512 \times 832$ & 17039360 & 2469945 & 6.8987 \\
\hline \multirow[t]{3}{*}{$\mathrm{HD}(720 \times 1280)$} & 4 & $720 \times 1280$ & 36864000 & 4043969 & 9.1158 \\
\hline & 8 & $736 \times 1280$ & 37683200 & 4167967 & 9.0411 \\
\hline & 16 & $704 \times 1280$ & 36044800 & 4200327 & 8.5814 \\
\hline
\end{tabular}

Table 2. Analysis using color image coding

\begin{tabular}{cccccc}
\hline Frame size & SIB & Video size & N.B & N.C.B & C.R \\
\hline QCIF(144x176) & 4 & $144 \times 176$ & 405504 & 178218 & 2.2755 \\
& 8 & $160 \times 192$ & 491520 & 213508 & 2.3021 \\
CIF (288x352) & 16 & $128 \times 192$ & 393216 & 174727 & 2.2763 \\
& 4 & $288 \times 352$ & 1622016 & 559113 & 2.9011 \\
WVGA(480x800) & 8 & $288 \times 352$ & 1622016 & 560453 & 2.8941 \\
& 16 & $320 \times 384$ & 1966080 & 648312 & 3.0326 \\
HD(720x1280) & 8 & $480 \times 800$ & 6144000 & 1483717 & 4.141 \\
& 16 & $480 \times 800$ & 6144000 & 1485484 & 4.136 \\
& 4 & $512 \times 832$ & 6815744 & 1627941 & 4.1867 \\
& 16 & $720 \times 1280$ & 6815744 & 1927941 & 4.1867 \\
& $736 \times 1280$ & 15073280 & 2901033 & 5.1958 \\
\hline
\end{tabular}

Table 3. Analysis using real time image

\begin{tabular}{cccccc}
\hline Frame size & $\begin{array}{c}\text { I.B.S(internal } \\
\text { block size) }\end{array}$ & Video size & N.B & N.C.B & C.R \\
\hline QCIF(144x176) & 4 & $144 \times 176$ & 405504 & 177662 & 2.2824 \\
& 8 & $160 \times 192$ & 491520 & 213749 & 2.2995 \\
CIF (288x352) & 16 & $128 \times 192$ & 393216 & 171722 & 2.2898 \\
& 4 & $288 \times 352$ & 1622016 & 559065 & 2.9013 \\
& 8 & $288 \times 352$ & 1622016 & 56028 & 2.895 \\
WVGA(480x800) & 16 & $320 \times 384$ & 1966080 & 649744 & 3.0259 \\
& 4 & & & & 4.1332 \\
HD(720x1280) & 8 & $480 \times 800$ & 6144000 & 1486490 & 4.1266 \\
& 16 & $480 \times 800$ & 6144000 & 1488890 & 4.1902 \\
& 8 & $512 \times 832$ & 6815744 & 1626605 & 5.1535 \\
\end{tabular}

The study outcome of the proposed system has been subjected to comparative analysis where the outcome of proposed system has been compared with frequently exercised data hiding techniques as shown in Figure 3 i.e. Histogram modification, difference expansion, and least significant bits with respect to new performance parameters. It should be noted that majority of the existing scheme is about compressing

Framework for reversible data hiding using cost-effective encoding system for ... (Manjunath Kamath K.) 
the size of the signal while less emphasis is given on the retention of quality signal. However, proposed system offers better to embedding time, extraction time, Peak Signal-to-Noise Ratio, and Embedding Capacity apart from signal quality.

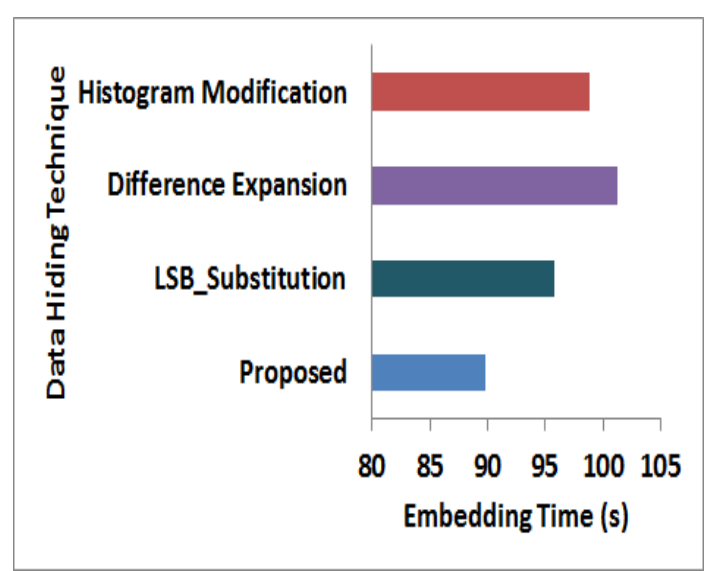

(a)

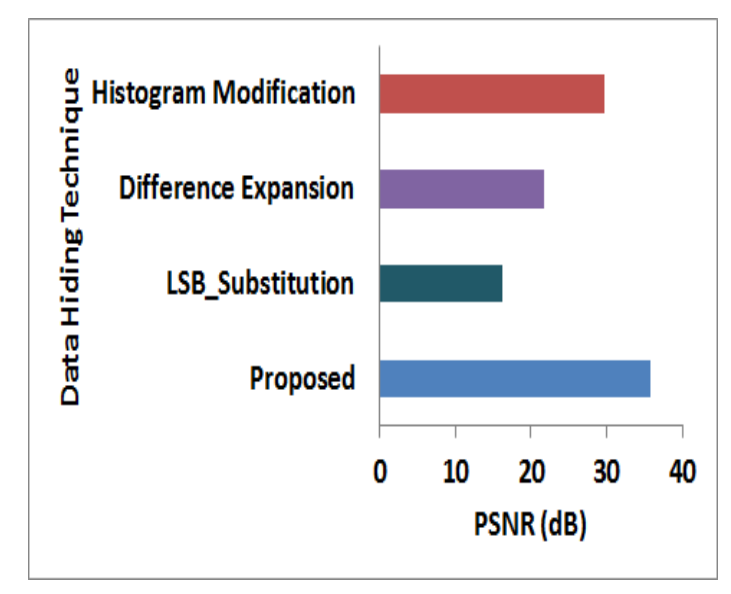

(c)

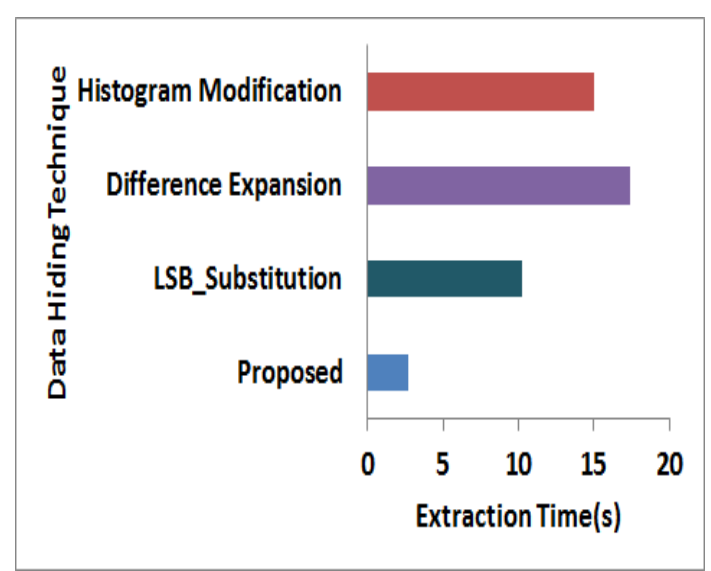

(b)

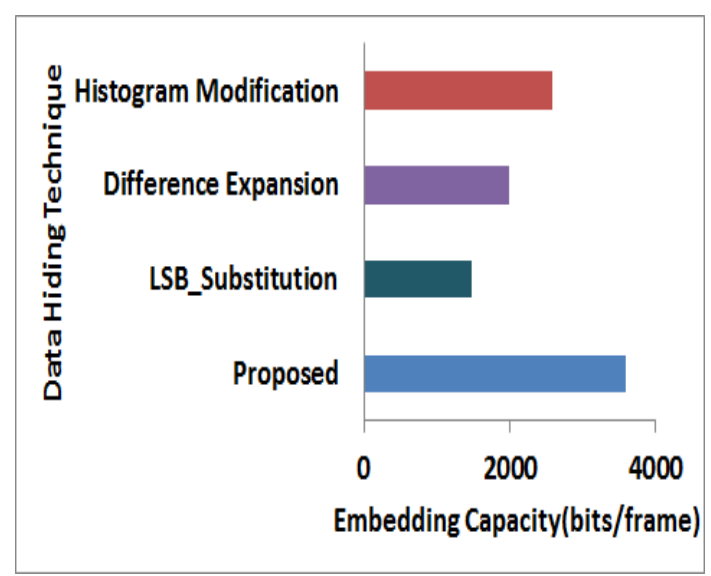

(d)

Figure 3. Performance comparative analyses, (a) embedding time, (b) extraction time, (c) psnr, (d) embedding capacity

The study outcome shows that proposed system excels better performance in every respect in contrast to existing system. From embedding and extraction time, difference expansion is found to have an inferior performance because of its bit-replacement of its gray scale pixels. Even histogram modification approach also suffers from a problem of limited capacity that leads to maximized embedding and extraction time. On the other hand, LSB based approach offers benefits as it reduces data degradation however such data could get lost. From PSNR viewpoint, proposed system is always found to offer better performance in contrast to existing system. It is found that existing system suffers from problems of retention of round off errors as well as higher number of searches. On the other hand, proposed system offers an extensive encoding process where a tree-based scheme has been used for comprehensive improvement in the embedding capacity. At the same time, the adoption of larger block size results in better quality of the video as well as increased payload in the receiver end. The overall memory consumption is quite very less and is within a permissible limit for any application working over real time. Hence, the proposed system offers a cost-effective encoding mechanism in the form of reversible data hiding with good balance between the security standard and data quality over the signal end. 


\section{CONCLUSION}

Basically, reversible data hiding demands exact recovery of secret message. The analysis of carried out considering cover data as video and secret data as image. This is proven by showing the number of bits and compressed bits (for secret image). This proposed system has introduced a unique reversible data hiding scheme with cost effective implementation method. The prime motive of the proposed concept is to prove the prevalent myth that reversible data hiding is quite a challenging part to implement owning to its complexities associated with uncompressed data. Irrespective of presence of different existing data hiding schemes that mainly uses compression-based approach, they cannot ensure the reversibility factor of the cover data (video) and this is mainly due to the lossy characteristic of the adopted standard of video compression. Therefore, the proposed reversible data hiding scheme make use of I-frame as well as P-frame for accomplishing the reversibility of the bitstream. Basically, a complete picture is represented as I frame and only the altered part of the image is considered as $\mathrm{P}$ frame. As the proposed system uses blocking that initiates from the I-frame and uses P-frame for prediction purpose of its prior frame in order to perform more size reduction. Hence, it can be said that both I and P frame achieves reversibility. A significant increase in the payload over the embedding capacity has been found with significant complexity reduction.

\section{REFERENCES}

[1] R. Zaheer et al., "A literature survey on various approaches of data hiding in images," 2017 International Conference on Innovations in Information, Embedded and Communication Systems (ICIIECS), Coimbatore, pp. 1-5, 2017.

[2] R. Abbasi, et al., "A new multilevel reversible bit-planes data hiding technique based on histogram shifting of efficient compressed domain," Vietnam Journal of Computer Science, vol. 5, no. 2, pp. 185-196, 2018.

[3] M. T. Ahvanooey, et al., "AITSteg: An Innovative Text Steganography Technique for Hidden Transmission of Text Message via Social Media,” IEEE Access, vol. 6, pp. 65981-65995, 2018.

[4] A. K. Singh, et al., "Recent trends in multimedia data-hiding: a reliable mean for secure communications," Journal of Ambient Intelligence and Humanized Computing, vol. 11, pp. 1795-1797, 2019.

[5] X. Zhang, et al., "Lossless and Reversible Data Hiding in Encrypted Images With Public-Key Cryptography," in IEEE Transactions on Circuits and Systems for Video Technology, vol. 26, no. 9, pp. 1622-1631, Sep. 2016.

[6] R. Abbasi, et al., "Efficient Lossless Compression Based Reversible Data Hiding Using Multilayered n-Bit Localization," Security and Communication Networks, pp. 1-13, 2019.

[7] Rathika R. and S. Kumaresan, "Survey on reversible data hiding techniques,"2016 3rd International Conference on Advanced Computing and Communication Systems (ICACCS), Coimbatore, pp. 1-4, 2016.

[8] X. Li, et al., "A brief review on reversible data hiding: Current techniques and future prospects," 2014 IEEE China Summit \& International Conference on Signal and Information Processing (ChinaSIP), Xi'an, pp. 426-430, 2014.

[9] J. Lakshmi and J. Rajeshwari, "Review on safe reversible image data hiding across an enciphered environment," 2017 International Conference on Computing Methodologies and Communication (ICCMC), Erode, pp. 22-26, 2017.

[10] Kamath, K. Manjunath, and R. Sanjeev Kunte, "Framework for Data Hiding Operation Using Motion Vectors for Effective Imperceptibility Performance," In International Conference on Computer Networks and Inventive Communication Technologies, Springer, Cham, pp. 9-20, 2019.

[11] H. Wang, et al., "Reversible AMBTC-Based Data Hiding With Security Improvement by Chaotic Encryption," in IEEE Access, vol. 7, pp. 38337-38347, 2019.

[12] H. Wu, et al., "Reversible Data Hiding With Image Contrast Enhancement Based on Two-Dimensional Histogram Modification," in IEEE Access, vol. 7, pp. 83332-83342, 2019.

[13] F. Peng, et al., "Reversible Data Hiding in Encrypted 2D Vector Graphics Based on Reversible Mapping Model for Real Numbers," in IEEE Transactions on Information Forensics and Security, vol. 14, no. 9, pp. 2400-2411, Sep. 2019.

[14] P. Puteaux and W. Puech, "An Efficient MSB Prediction-Based Method for High-Capacity Reversible Data Hiding in Encrypted Images," in IEEE Transactions on Information Forensics and Security, vol. 13, no. 7, pp. 1670-1681, Jul. 2018.

[15] Z. Qian, et al., "New Framework of Reversible Data Hiding in Encrypted JPEG Bitstreams," in IEEE Transactions on Circuits and Systems for Video Technology, vol. 29, no. 2, pp. 351-362, Feb. 2019.

[16] S. Yi and Y. Zhou, "Separable and Reversible Data Hiding in Encrypted Images Using Parametric Binary Tree Labeling," in IEEE Transactions on Multimedia, vol. 21, no. 1, pp. 51-64, Jan. 2019.

[17] L. Xiong, et al., "High-Capacity Reversible Data Hiding for Encrypted Multimedia Data With Somewhat Homomorphic Encryption," in IEEE Access, vol. 6, pp. 60635-60644, 2018.

[18] R. Jiang, et al., "Reversible Data Hiding in Encrypted Three-Dimensional Mesh Models," in IEEE Transactions on Multimedia, vol. 20, no. 1, pp. 55-67, Jan. 2018.

[19] S. Xiang and X. Luo, "Reversible Data Hiding in Homomorphic Encrypted Domain by Mirroring Ciphertext Group," in IEEE Transactions on Circuits and Systems for Video Technology, vol. 28, no. 11, pp. 3099-3110, Nov. 2018.

[20] W. Zhang, et al., "Reversible Data Hiding in Encrypted Images by Reversible Image Transformation," in IEEE Transactions on Multimedia, vol. 18, no. 8, pp. 1469-1479, Aug. 2016.

[21] B. Ma and Y. Q. Shi, "A Reversible Data Hiding Scheme Based on Code Division Multiplexing," in IEEE Transactions on Information Forensics and Security, vol. 11, no. 9, pp. 1914-1927, Sep. 2016. 
[22] Z. Qian and X. Zhang, "Reversible Data Hiding in Encrypted Images With Distributed Source Encoding," in IEEE Transactions on Circuits and Systems for Video Technology, vol. 26, no. 4, pp. 636-646, Apr. 2016.

[23] Z. Qian, et al., "Separable Reversible Data Hiding in Encrypted JPEG Bitstreams," in IEEE Transactions on Dependable and Secure Computing, vol. 15, no. 6, pp. 1055-1067, 2018.

[24] X. Cao, et al., "High Capacity Reversible Data Hiding in Encrypted Images by Patch-Level Sparse Representation," in IEEE Transactions on Cybernetics, vol. 46, no. 5, pp. 1132-1143, May 2016.

[25] Y. Qiu, et al., "Adaptive Reversible Data Hiding by Extending the Generalized Integer Transformation," in IEEE Signal Processing Letters, vol. 23, no. 1, pp. 130-134, Jan. 2016.

[26] I. F. Jafar, et al., "Efficient reversible data hiding using multiple predictors," The Computer Journal, vol. 59, no. 3, pp. 423-438, 2016.

[27] X. Hu, et al., "Minimum Rate Prediction and Optimized Histograms Modification for Reversible Data Hiding," in IEEE Transactions on Information Forensics and Security, vol. 10, no. 3, pp. 653-664, Mar. 2015.

[28] X. Li, et al., "Efficient Reversible Data Hiding Based on Multiple Histograms Modification," in IEEE Transactions on Information Forensics and Security, vol. 10, no. 9, pp. 2016-2027, Sep. 2015.

[29] J. Zhou, et al., "Secure Reversible Image Data Hiding Over Encrypted Domain via Key Modulation," in IEEE Transactions on Circuits and Systems for Video Technology, vol. 26, no. 3, pp. 441-452, Mar. 2016.

[30] "YUV Video Sequences," Arizona State University, [Online], Available: http://trace.kom.aau.dk/yuv/index.html, [Retrived on 06-05-2020].

\section{BIOGRAPHIES OF AUTHORS}

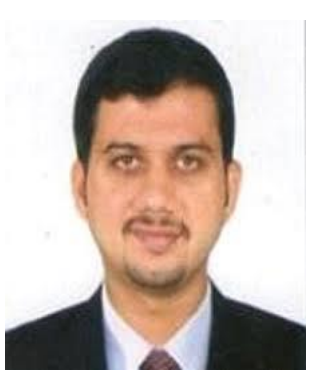

Mr. Manjunath Kamath K received M.Tech degree from Visvesvaraya Technological University. He is currently working as a Assistant Professor in the Department of Information Science and Engineering, Yenepoya Institute of Technology, Moodbidri, Karnataka. He has professional membership of ISTE. His research interests include information security, image, and video processing.

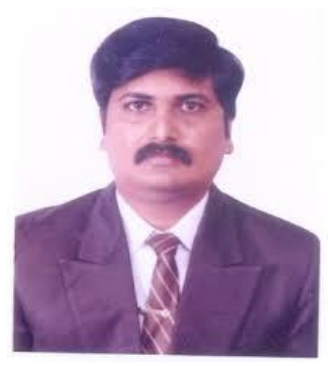

Dr. R Sanjeev Kunte received M.Tech degree from Visvesvaraya Technological University and Ph.D from University of Mysore in 2009. He is currently working as a Professor in the Department of Computer Science and Engineering, J.N.N College of Engineering, Shivamogga, Karnataka. He has professional membership of ISTE, IETE. He has published many papers in reputed conferences/journals. His research area includes information security, pattern recognition and image processing. 